NASA Technical Memorandum 88960

\title{
Identification of Structural Interface Characteristics Using Component Mode Synthesis
}

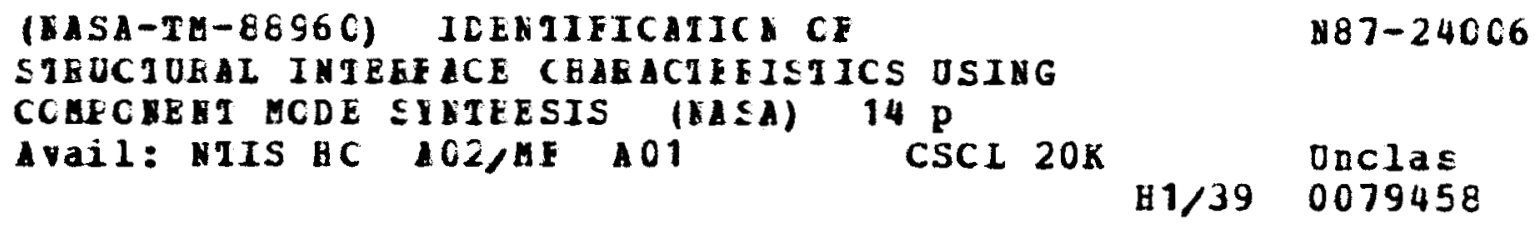

A.A. Huckelbridge

Case Western Reserve University

Cleveland, Ohio

and

C. Lawrence

Lewis Research Center

Cleveland, Ohio

Prepared for the

Vibrations Conference

sponsored by the American Society of Mechanical Engineers

Boston, Massachusetts, September 27-30, 1987 
IOENTIF ICATION OF STRUCTURAL INTERFACE CHARACTERISTICS USING COMPONENT MODE SYNTHESIS

\author{
A. A. Huckelbridge \\ Case Western Reserve University \\ Cleveland, Ohio 44106
}

and

C. Lawrence

National Aeronautics and Space Administration

Lewi s Research Center

Cleveland, Ohio 44135

\section{INTRODUCTION}

The dynamic response of large structural systems is often analyzed using component mode synthesis (CMS) techniques. CMS is widely accepted for predicting coupled system response with increased modeling efficiency and flexibility over conventional methods. CMS

$\stackrel{\omega}{\stackrel{F}{F} \text { techniques utilize a reduced set of component modes }}$ w the inability to adequately model the connections between components has limited the application of CMS. Connections between structural components, and between components and ground are often mechanically complex and difficult to accurately model analytically. The modeling of these connections can profoundly influence predicted system behavior. This is because only the connections determine the boundary conditions which are imposed upon the system components. Thus, improved analytical models for connections are needed to extend the applicability of CMS and to improve system dynamic predictions.

Parameter identification (PID) techniques can be used to improve predicted response when experimental data are available. Modeling accuracy is improved with PID by reducing discrepancies between the measured characteristics of a physical system with those predicted by an analytical model of the system. Many techniques are available to carry out this process of parameter refinement. Most involve the determination of a set of structural parameters which optimally minimize differences between experiment and analytical prediction.

This study explores combining CMS and PID methods to improve the analytical modaling of the connections in a component mode synthes is model. The approach involves modeling components with either finite elements or experimental modal data and then joining the components with physical connecting elements at their interface points. Interface connections in both the translational and rotational directions are addressed. once the system model is derived, experimentally measured data is used with PID methods to improve the characterizations of the connections between components. Corrections in the connection properties are computed in terms of physical parameters. With this approach, the physical characteristics of the connections can be better understood, in addition to providing improved input for the CMS model.

The identification of connection characteristics is simplified by requiring individual components to be verified before they are incorporated into the coupled system model. This requirement will normally not present any difficulties, since component testing and verification has become a regular practice. With this requirement, the components are verified before they are used in the coupled system model. Any differences between the measured and predicted coupled system response can be solely attributed to inaccuracies of the estimated properties of the connections. Also, the quantity of test data that must be obtained from the coupled system is greatiy reduced. This is particularly useful when it is impractical to obtain a complete set of vibration test data for a coupled structure. Examples, include large space structures, spacecraft systems, and turbomachinery.

Component Coupling Procedure

Numerous variations of the CMS method are currently available for the dynamic analysis of coupled structural systems ( 1 to $\underline{3}$ ). In the classical CMS approach, all of the system components are characterized in the modal domain using their respective modal parameters (frequencies and mode shapes). Coupling between components also is performed in the modal domain through use of modal constraints. These constraints are derived from displacement compatibility conditions existing at the component interface locations. With the classical CMS approach, any components or connections that have been modeled in terms of physical coordinates (e.g., finite elements) must be transformed into the modal domain before they can be included in the coupled system equations of motion. The system equations, in terms of modal coordinates, are used to compute the system natural frequencies. The system mode shapes are computed by transforming the mode shapes obtained from the system equations back to physical coordinates.

Recent applications of the CMS method have shifted from the classical approach of utilizing only modal coordinates. Instead, techniques that use a mixture of both modal and physical coordinate systems have been implemented (3). There are several reasons for the shift to a "mixed" coordinate set. One reason is that a combination of component types can be incorporated into the coupled system equations without requiring all of the components to be in identical coordinate systems. This is particularly useful when some of the components have been modeled using $F . E$. methods and other component models have been derived from modal test data. In most of the currently used CMS methods boundary degrees of freedom of all of the components are expressed in terms of physical coordinates, and the internal degrees of freedom are expressed in either modal or physical coordinates. The inherent efficiency of the component representation is retained. With physical boundary coordinates, components can be coupled utilizing classical direct stiffness assembly techniques as in conventional F.E. computer codes. Furthermore, nonlinear connecting elements can be used when boundary degrees of freedom are in physical coordinates. In the classical CMS approach, where modal coordinates are used, it is very difficult to incorporate nonlinearities into the coupled system model because of the difficulties associated with defining modal parameters for nonlinear elements. 
This study develops a simplified variation of the previously mentioned procedures for CMS. The procedure is defined to be compatible with PID procedures which will be used subsequently for identifying the component interface characteristics. The modal components are first converted to "pseudo" finite elements to connect modal components to physical finite element components. The pseudo elements are then treated in the same manner as conventional finite elements, i.e., system property matrices are assembled through direct stiffness techniques.

Consider the system shown in $\mathrm{Fig}$. 1. This system is comprised of two components which are coupled by a physical connecting component. The undamped, free vibration equation of motion for the uncoupled system is written in terms of physical coordinates as:

$$
\left.\left[\begin{array}{lll}
{\left[M^{I}\right]} & & \\
& {[0]} & \\
& & {\left[M^{I I}\right]}
\end{array}\right]\left\{\begin{array}{l}
\ddot{u}^{I} \\
\ddot{u}^{c} \\
\ddot{u}^{I I}
\end{array}\right\}+\left[\begin{array}{lll}
{\left[k^{I}\right]} & & \\
& {\left[k^{c}\right]} & \\
& & {\left[k^{I I}\right]}
\end{array}\right]\left\{\begin{array}{l}
u^{I} \\
u^{c} \\
u^{I I}
\end{array}\right\}=10\right\}
$$

where [M] and [K] are the component system mass and stiffness matrices, and $\{\ddot{u}\}$ and $\{u\}$ are the vectors of component nodal accelerations and displacements (the superscripts refer to the component identification). Equation (1) can further be partitioned by separating displacements internal to the components from those that are at the interfaces between components. When this is done Eq. (1) is written as:

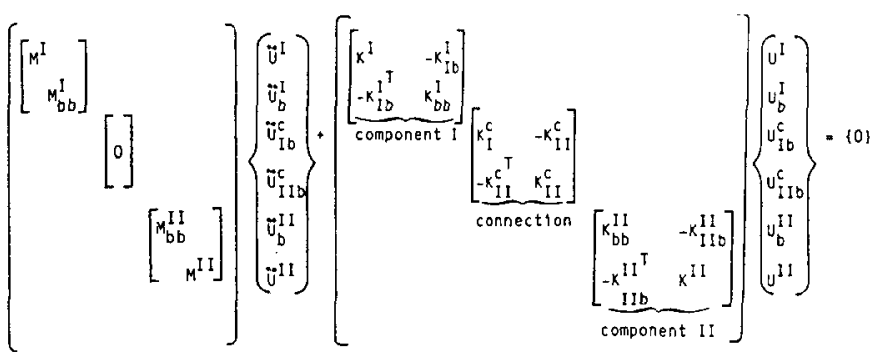

The coupled system equation is obtained by applying the displacement compatibility conditions at the interface between the components and the connections. The displacements of the component and the connection must be equal at the interface therefore:

$$
U_{b}^{I}=U_{I b}^{C}
$$

and

$$
U_{b}^{I I}=U_{I I b}^{c}
$$

Using Eq. (3), the transformation matrix, [T], which relates the dependent and independent displacement sets is:

$$
\left\{\begin{array}{c}
U^{I} \\
U_{b}^{I} \\
U_{I b}^{c} \\
U_{I I b}^{c} \\
U_{b}^{I I} \\
U^{I I}
\end{array}\right\}=\left[\begin{array}{cccc}
1 & 0 & 0 & 0 \\
0 & 1 & 0 & 0 \\
0 & 1 & 0 & 0 \\
0 & 0 & 1 & 0 \\
0 & 0 & 1 & 0 \\
0 & 0 & 0 & 1
\end{array}\right\}\left\{\begin{array}{c}
U^{I} \\
U_{b}^{I} \\
U_{b}^{I I} \\
U^{I I}
\end{array}\right\}
$$

From conservation of energy principles and the above transformation, the coupled equation of motion is found from:

$$
[K]_{\text {coupled }}=[\mathrm{T}]^{\mathrm{T}}[\mathrm{K}]_{\text {uncoupled }}[\mathrm{T}]
$$

and

$$
[M]_{\text {coupled }}=[T]^{\top}[M]_{\text {uncoupled }}[T]
$$

Substituting the property matrices from Eq. (2) into $\mathrm{Eq}$. (5) the coupled equation of motion is:

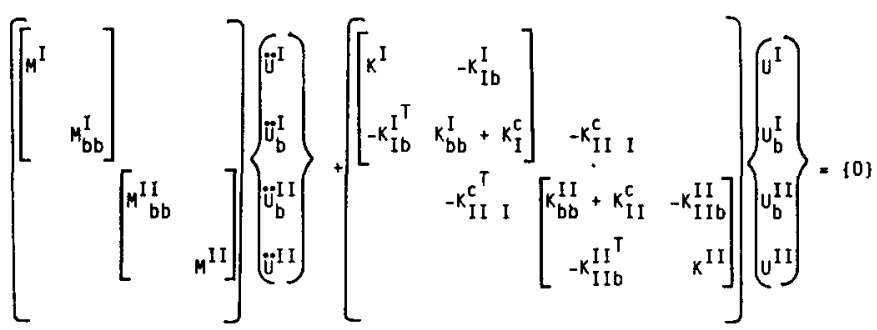

The procedure outlined above can be used to couple any number of physical components into the system equations. As mentioned previously, when modal components are to be coupled into the system model they are converted to pseudo physical components and then are treated in the same manner as conventional physical components. The pseudo physical property matrices are obtained from orthogonality relationships between the property matrices and the modal parameters. When the component mode shapes are normalized so that the modal mass matrix equals the identity matrix, the modal and physical property matrices are related by:

$$
[\phi]^{\top}[M][\phi]=[\mathrm{I}]
$$

and

$$
[\phi]^{\top}[K][\phi]=\left[-W^{2}\right]
$$

where [M] and [K] are the component physical mass and stiffness matrices, [ $\left.w^{2}-\right]$ are the component frequencies, and $[\phi]$ is the matrix of component mode shapes. 
When experimental modal data is used to characterize the component, the matrix $[\phi]$, containing the component mode shapes may be rectangular. If "m" mode shapes are measured, and the value of the mode shapes are recorded at " $n$ " different physical locations on the component, then the mode shape matrix will be of order $n \times m$. Normally, there will be more measurement locations available than there will be modes that $c$ an be measured. To obtain a square modal matrix from experimental mode shape data, data at some measurement points can be neglected so that the number of points is equal to the number of modes. When data at measurement points is discarded no information is lost as far as the overall system response is concerned, so long as measurements at the component's interface points are retained. Once a square mode shape matrix is available, the pseudo physical property matrices are related to the modal data by:

$$
\left[M_{p}\right]=\left[\phi^{\top}\right]^{-1}[\phi]^{-1}
$$

and

$$
\left[K_{p}\right]=\left[\phi^{\top}\right]^{-1}\left[-w^{2}-\right][\phi]^{-1}
$$

where $\left[M_{p}\right]$ and $[K p]$ are the component pseudo mass and stiffness matrices. (The coefficients of the mass and stiffness matrices are in terms of physical coordinates corresponding to the location and direction where the mode shapes are measured).

The matrices computed in Eq. (8) are designated as pseudo matrices because their physical interpretation is unlike that of conventional mass and stiffness matrices. Because it is impractical to measure all of the component modes, the modal data will be incomplete (see (4)) and will not contain all the information required to produce the actual component mass and stiffness matrices. Therefore, although the mass and stiffness matrices computed in $\mathrm{Eq} .(8)$ are in terms of physical rather than modal coordinates, the matrices will not necessarily represent the actual physical mass and stiffness characteristics of the component. The mass and stiffness matrices from Eq. (8) will reproduce the measured frequencies and mode shapes, and will be suitable for representing the component in the coupled system model.

Either the "free" or the "fixed" boundary component modes can be used for the component characterization. The "free" mode shapes are those modes that correspond to the component when it is in the unconstrained or free boundary condition. In many situations these modes are more conveniently obtained than the fixed boundary modes. This is particularly true when the modes are measured experimentally, because the component itself does not have to be physically constrained during the experimental testing. In practice, the free boundary condition often is approximated by suspending the component from flexible cords or by supporting it on soft springs.

The fixed modes are obtained by simultaneously constraining all of the component's boundary degrees of freedom while performing the modal testing. Analytically, the fixed modes are computed as easily as the free modes. Experimentally, they are more difficult to obtain, because all of the component's boundary degrees of freedom must be fully constrained during the experiment. To attain this condition requires that elaborate fixtures be attached at the components boundary locations, and in practice, full constraint is never completely achieved. Another difficulty of using fixed boundury mode shapes is that an additional set of "static" deflection or constraint modes must be added to the set of fixed boundary modes. These modes are required so that the component will have flexibility at its boundary locations where it is connected to adjacent components.

Normally, the values of the experimental mode shapes are measured in the translational directions. It is not generally practical to measure the values of the mode shapes in the rotational directions because of limitations in available instrumentation. However, it is sometimes desirable to couple rotational degrees of freedom between components. If the values of the mode shapes are not measured in the rotational directions, the pseudo matrices will only have translational degrees of freedom and there will not be means of coupling the rotational connecting stiffnesses. To circumvent this difficulty, the rotational values of the mode shapes can be extrapolated from the translational values, either by curve fitting through the translational degrees of freedom and then computing the slope of the curve at the connection location, or by using an approximate F.E. model of the component (see [(]).

When the rotational values are extrapolated from a curve fit any existing rotational inertia effects will not be reflected in the values of the rotations. Neglecting the actual independent motion of the rotation implies that there is no rotational inertia and that the rotations are dependent on the translations. Because of this dependence, the combined translational/ rotational mode shapes can not be used directly to compute the pseudo matrices without encountering numerical problems during the matrix inversions in Eq. (8). A solution to this difficulty is to initially use only the translational mode shapes to compute the pseudo matrices. Then, a transformation which is based on the dependence between the rotations and translations is used to transform the pseudo matrices from the translational coordinate system to a combined translational/rotational system.

The dependent rotational values of the mode shapes can be related to the independent translations by:

$$
U_{\theta_{j}}=\sum_{i}^{n} a_{i} U_{\Delta i}
$$

Where $U_{\theta_{j}}$ is the dependent rotation at $j, U_{\Delta i}$ are

the translations at the independent measurement points, $a_{i}$ are the coefficients relating the independent translations to the dependent rotations (determined from curve fit, etc.), and $n$ is the number of independent measurement points.

The transformation from the mixed coordinate matrices to the entirely translational pseudo property matrices is: 


$$
\left\{U_{\Delta}\right\}_{n}=\left[\begin{array}{ll}
T^{\prime} & 0 \\
0 & I
\end{array}\right]_{n \times n}\left\{\begin{array}{l}
U_{\theta} \\
U_{\Delta}^{\prime}
\end{array}\right\}_{n}
$$

where $\left[T^{\prime}\right]$ is the transformation matrix derived from the relationships in Eq. (9) and $\left\{u_{\Delta}\right\}^{\prime}$ is a subset of $\left\{u_{0}\right\}$. For each rotational degree of freedom that is added in $\left\{u_{\theta}\right\}$, a translational degree of freedom is removed from $\left\{u_{\Delta}\right\}^{\prime}$. The selection of the trans1 ational degrees of freedom that are removed is arbitrary, and since a translation is removed for each rotation that is added, both systems will contain the same number of degrees of freedom.

Using the original translational pseudo property matrices from Eq. (8), the transformation in Eq. (10), dnd principles of cunservation of energy, the pseudo matrices are derived in the combined translational/ rotational coordinate system by:

$$
\begin{aligned}
& {\left[K_{p}\right]_{U_{\Delta}^{\prime} U_{\theta}}=\left[\begin{array}{ll}
T & 0 \\
0 & I
\end{array}\right]^{\top}\left[\begin{array}{l}
K_{p} \\
]_{U_{\Delta}}
\end{array}\right]\left[\begin{array}{ll}
T & 0 \\
0 & I
\end{array}\right]} \\
& {\left[M_{p}\right]_{U_{\Delta}^{\prime} U_{\theta}}=\left[\begin{array}{ll}
T & 0 \\
0 & I
\end{array}\right]^{\top}\left[M_{p}\right]_{U_{\Delta}}\left[\begin{array}{ll}
T & 0 \\
0 & I
\end{array}\right]}
\end{aligned}
$$

Once the component pseudo matrices in Eq. (11) are computed, they can be inserted into the system equations of motion and coupled to adjacent components using the previously discussed procedures.

The final coupled system equations can be used to predict the overall system dynamic characteristics. The frequencies that are computed from this equation will correspond to the overall system resonances. The accuracy of the predicted frequencies will be dependent on the precision with which the connections between components have been modeled. It has been assumed that the component modal models have been verified and are accurate, and also, that the proper component modes have been included in the model to adequately predict system response (see sample problem one).

The mode shapes derived from the system equations will correspond to the physical degrees of freedom included in the system model. When the combined translational/rotational model is used some of the mode shape values will correspond to translational degrees of freedom and some to rotations. The accuracy of the mode shapes, like the frequencies, will be dependent on the adequacy of the component modal representations and the modeling of the connections.

\section{Parameter Identification Procedure}

Once the system equations of motion and their corresponding frequencies and mode shapes are computed, and the experimental system modes have been measured, PID $c$ an be used to find an improved set of connection parameters that better predict the measured experimental system data. For this study the Weighted Least Squares method for parameter estimation is used (6).

If $\{\bar{c}\}$ and $\{c\}$ are vectors containing the measured and computed system frequencies and mode shapes respectively, then the weighted squared difference between the predicted and measured characteristics is:

$$
\{F\}=[W](\{\bar{C}\}-\{C\})^{2}
$$

where $[W]$ is the weighting matrix and $\{F\}$ is a vector of weighted squared differences. To find the set of connection parameters that minimizes the weighted squared differences, the derivative of $\{F\}$ with respect to the connection parameters is set to zero. Noting that the predicted characteristics $\{c\}$, are a function of the connection parameters $\{r\}$, the derivative of $\{F\}$ is written as:

$$
\frac{\partial\{F\}}{\partial\{r\}}=[W](\{\bar{c}\}-\{c\}) \frac{\partial\{c\}}{\partial\{r\}}=\{0\}
$$

Expanding (c) in a Taylor series and truncating higher order terms, $\{c\}$ is approximated as:

$$
\{c\}=\{c\} \text { est }+\frac{\partial\{c\}}{\partial\{r\}}\{\Delta r\}
$$

Where $\{\Delta r$ ) are the differences between the estimated and actual values for the connection parameters. Substituting Eq. (14) into Eq. (13) and letting $a\{c\} / \partial\{r\}=[S]$ leads to:

$$
[W](\{\bar{c}\}-\{C\} \text { est }-[S]\{\Delta r\})[S]=\{0\}
$$

From Eq. (15) it is desired to solve for $\{\Delta r\}$ so that the actual connection parameters can be determined. Solving for $\{\Delta r\}$ can not be accomplished by simple inversions, however, because in general the number of measured and predicted characteristics will be greater than the number of connection parameters, rendering the matrix [S] to be nonsquare. The vector $\{\Delta r\}$ can be solved for if Eq. (15) is first premultiplied by $[S]^{\top}$. When this is done, $\{\Delta r\}$ is solved as:

$$
\{\Delta r\}=\left([S]^{\top}[W][S]\right)^{-1}[S]^{\top}[W]\left(\{\bar{c}\}-\{c\}_{E S T}\right)
$$

An updated set of connection parameters is computed by:

$$
\{r\}=\{r\}_{E S T}+\{\Delta r\}
$$

or by substituting from Eq. (16):

$\{r\}=\{r\}_{E S T}+\left([S]^{\top}[W][S]\right)^{-1}[S]^{\top}[W]\left(\{\bar{c}\}-\{c\}_{E S T}\right)$

Since $\{c\}$ is approximated by a truncated series, the improved connection parameters will be only an approximation to the final parameters. However, the final parameters $c$ an be obtained by iterating on Eq. (18).

A direct approach for computing the elements of the sensitivity matrix [S] is to perturb the analytical model with changes in the connection parameters, and then compute the resulting changes in the system characteristics. The elements are then computed by setting $T i j$ equal to the change in the $\mathrm{Ci}$ characteristic divided by the change in the $r j$ connection parameter. Alternative methods for computing these derivatives have been presented (see (7)) but for problems such as the example, with only a small number of connection parameters, the above method is adequate.

The selection of the system characteristics that are used in the estimation procedure is determined by data acquistion capability. Experimentally, it is generally easier to measure frequencies than mode shapes, so in many cases it may be practical to include more frequencies than modes shapes. Characteristics 
other than frequencies and mode shapes also can be utilized; in (8), it is suggested that kinetic energy may be a usefuT characteristic. Once the characteristics are chosen, the weight that is placed on each characteristic must be determined. If one characteristic is measured more accurately than another, then it can be weighted more heavily.

When the number of system characteristics is large, the size of the weighting and sensitivity matrices increases, and the matrix in Eq. (18) may become ill conditioned for inversion (see (9)). The PID procedure only requires a minimum number of system characteristics to adequately identify the connection parameters since each component has already been verified. Therefore, the size of the matrices in Eq. (18) will be kept small and inversion problems will be minimized. Another problem may arise when the analytical model cannot be exactly made to $f$ it the experimental data. When this is the situation the set of connection parameters that minimizes the differences, rather than eliminates them, must be used. The model may not be able to produce the desired measured system characteristics because of limitations in the component modal representation. Also, if the experimentally measured modes are not orthogonal, perfect agreement $c$ an never be achieved because the analytical model can only produce orthogonal mode shapes.

\section{Sample Problem One: Coupled Beams}

The following sample problem is offerred to demonstrate the component coupling and parameter identification procedures. To verify these procedures simulated experimental data generated from a F.E. model was used. The sample problem (Fig. 2) is comprised of two simply supported beams connected at their ends. For simplicity, both beam components were made identical. In actual applications the system can be partitioned into any set of components that is desired. Each of the components in this problem are discretized into seven massless, planar beam elements. Concentrated translational masses are added between the elements at nodes 2 through 7 and 10 through 15. The components are connected by a rotational spring ( $K=10 . E 5)$ at nodes eight and nine. A connection also is made to ground by a rotational spring $(K=10 . E 5)$ added to the second component at node 16 .

The accuracy of the computed system frequencies as a function of the number of modes used for the component representations was evaluated with six, four, and two component modes (see Table l). Both the six and four component mode representations produced system frequencies that are in good agreement with the baseline F.E. solution. Although there are only six component modes in the F.E. solution, the six mode representation does not produce exact frequencies because the F.E. model has more than 6 degrees of freedom. The two mode representation allows for the first and third modal frequencies to be predicted satisfactorily but does not provide enough information for an accurate prediction of the second and fourth frequencies. At least two component modes are required so that there will be a rotational degree of freedom at each end of the component that is connected to ground (only one mode is needed for the other component). In every case the component mode solution produced frequencies that are higher than the baseline frequencies. This is understandable since the component mode solution uses a truncated set of modes and therefore does not include all of the component's flexibility.
For the initial attempt at identifying the connection properties, only the simulated system frequencies from the F.E. model (Table II) were used in the parameter identification routines. It is preferable that the connection properties be identified without having to use system mode shapes because the mode shapes are considerably more difficult to experimentally measure than the frequencies. When either six or four component mode representations were used two possible solutions were found for the $K 1$ and $K 2$ connecting stiffnesses which satisfied the system frequency constraints (see Table II). The chosen solution was dependent on the initial starting estimates for $k 1$ and K2. Although neither solution is equal to the actual connecting stiffnesses, the first one is reasonably close considering the limited number of system data used and the approximation of the component modal representation. When either of the solutions are input into the F.E. model they produce system frequencies that are very close to the exact frequencies. The first solution does produce a better set of system mode shapes. In an actual application, without more than system frequency informution, it would be impossible to determine which of the two solutions is closer to the actual values of the connecting stiffnesses. Furthermore, since both the five and two system frequency cases produced similar solutions there is no advantage to using more than two system frequencies. When two component modes are used a maximum of four system frequencies are available, therefore the five system frequency case cannot be analyzed. For the two component modes and two system frequency case, the solution failed to converge.

A subsequent attempt, using a combination of both system frequencies and mode shapes was made with the expectation that the identification of the connection properties would be improved. By adding the first mode shape as a constraint, along with the first five system frequencies, the second multiple solution was eliminated. When only one system frequency and one mode shape was used, the problem still converged to the first solution regardiess of the initial estimates for the connecting stiffnesses. This combination of system data is ideal because, while it eliminates the multiple solution, it only requires a minimal amount of experimental data. Similar results were produced for both the six and four component mode representations, while the two mode representation continued to present difficu?ties.

\section{Sample Problem Two: RSD Rig Verification}

once the component coupling and parameter identification algorithms were evaluated with simulated data (Sample Problem One), it was decided to assess the procedures using actual experimental data. To accomplish this, the RSD (Rotating Structural Dynamics Rig) at NASA Lewis Research Center was selected. The RSD rig (Fig. 3 ) is designed to simulate engine structures to study active rotor control and system dynamics (component interaction) problems. The rig components, although considerably simpler than a real turbine engine's, were scaled such that they would simulate an actual engine's structural dynamics response characteristics.

The objective of the parameter identification was to determine the stiffnesses of the squirrel cage bearing support that connects each end of the rotor to the support frame. To accomplish this, the RSD rig was divided in two components; the rotor support frame, and the rotor. Each of these components was characterized verified experimentally, so that accurate component representations would be available for the 
coupled system model. In the system model the support frame was represented by an experimentally verified F.E. model while the rotor component was represented by experimental modal data. Since both components were experimentally verified, any differences that appeared between the predicted and measured system characteristics could be attributed to the uncertainties in the squirrel cage connections between components. This approach considerably simplified the verification task by reducing the quantity of modal data required from the coupled system.

The support frame finite element mesh is shown in Fig. 4. The frame is mounted on a relatively stiff base plate so grid points 35 through 39 are fully constrained. Grid points 19 and 20 , where the rotor is attached, were allowed to freely displace. This free condition is representative of the conditions used during the modal tests and is also compatibie with the requirements for the component coupling procedure. The grid points are connected with beam (bending and axial deformations) elements except for the diagonal elements at grid 35 which are modeled with rod (axial deformation only) elements. All of the elements are modeled with $A 36$ steel properties. The frame F.E. model was analyzed with NASTRAN, to compute the component frequencies and mode shapes ( $\mathrm{Fig} .5$ ). The frequencies were experimentally verified by using vibration data obtained from an HP 5423 Dynamic Analyzer. The rotor modal representation was obtained by measuring the rotor mode shapes in the free boundary condition. This condition was approximated by hanging the rotor from bungy cords. The component modal characteristics were generated from transfer function data obtained from the dynamic analyzer and impact testing. A total of six rotor modes were measured (see Fig. 6) including two rigid body and four elastic modes.

The support frame and rotor were coupled by combining the physical F.E. model of the frame with the modal representation of the rotor. For simplicity the coupled system model was constrained to motion only in the vertical plane. This restriction allowed for a reduction in the required number of degrees of freedom in the system model and allowed for all of the system testing to be performed in one plane. The coupled system frequencies for the six mode rotor representation are plotted along with the measured frequencies in Fig. 7. The predicted frequencies were computed for different values of squirrel cage stiffness to determine the effect that the cages have on the system frequencies. To generate these results it was assumed that both squirrel cages had identical stiffnesses. This was a rational assumption, since both cages are built to the same specifications. (Subsequent to this analysis the cage stiffness was measured as $5050 \mathrm{lb} / \mathrm{in}$. using a static loading test.)

Only the first three computed system frequencies are shown because only three frequencies were measured. When all three frequencies are used the cage stiffness is identified as $5750 \mathrm{lb} / \mathrm{in}$. This value is in good agreement with the measured stiffness (5050), considering that only three system frequencies were used for the parameter identification. In Fig. 7 it is shown that this amount of difference in cage stiffness does not have a significant effect on the system frequencies.

In addition to the six mode rotor representation, a four and two mode representation were used to determine the effect that the number of component modes has on the stiffness identification. The four mode representation identified the same cage stiffness as the six mode representation. The two mode representation identified the cage stiffness as about $2300 \mathrm{ib} / \mathrm{in}$. or only 46 percent of the measured stiffness. It was expected that the two mode representation would be insufficient for identifying the cage stiffness because this representation is inadequate for accurately predicting the system modes. It is obvious that the two mode representation cannot produce very good results because only rigid body modes are included in the representation, and the system modes involve elastic bending in the rotor. Although rules of thumb are available for determining the required number of modes, additional work is required in this area.

\section{CONCLUSION}

From the two sample problems analyzed in this study it was determined that the stiffness characteristics of component commections can be identified using component mode synthesis and parameter identification procedures. Furthermore, the characteristics can be identified using experimentally obtained component modal representation and a minimal quantity of measured system modal data. In the first sample problem it was found that multiple solutions are possible, but that they can be avoided when system mode shapes are included in the identification procedure. In the second problem it was found that the rotor for a rotor/support frame coupled system could be adequately represented by experimentally obtained modal data. It was also found that only three system frequencies had to be measured for the connection characteristics between the frame and rotor to be identified. From the results obtained thus far, it is determined that the quantity of data required for the component representations and for the connection characteristic identification is problem dependent. Therefore, each application must be treated on an individual basis.

\section{REFERENCES}

1. Hurty, W.C., 1964, "Dynamic Analysis of Structural Systems by Component Mode Synthes is, "JPL-TR-32-350, NASA CR-53057.

2. Craig, R.R. Jr., 1981, Structural Dynamics, John Wiley and Sons, New York.

3. Martinez, D.R., and Gregory, D.L., 1984, "A Comparison of Free Component Mode Synthes is Techniques Using MSC/NASTRAN," SAND 83-0025.

4. Berman, A., and Flannelly, W.G., 1971, "Theory of Incomplete Models of Dynamic Structures," AIAA Journal, $V_{01} .9$, No. 8, pp. 1481-1487.

5. Berman, A., and Nagy, E.J., 1982, Improvement of a Large Dynamic Analytical Model Using Ground Vibration Test Data," AIAA 23rd Structures, Structural Dynamics and Materials Conference, Part 1 , AIAA, New York, pp. 301-306.

6. Isenberg, J., 1979, "Progressing from Least Squares in Bayesian Estimation, " ASME Paper 79-WA/DSC-16.

7. Collins, J.0., Hart, G.C., Hasselman, T.K., and Kennedy, B., 1974, "Statistical Identification of Structures," AIAA Journal, Vol. 12, No. 2, pp. 185-190. 
Wada, B.K., Garba, J.A., and Chen, J.C., 1983,

Modal Test and Analys is Correlation, "Modal

Testing and Model Refinement, AMD-Vol. 59, D.F.H.

Chu, ed., ASME, New York, pp. 85-99.
9. Hasselman, T.K., 1983, "A Perspective on Dynamic Model Verification," Modal Testing and Model Refinement, AMD-Vol. 59, D.F.H. Chu, ed., ASME, New York, pp. 101-117.

TABLE 1. - COUPLED SYSTEM FREQUENCIES (SAMPLE PROBLEM ONE) [Connection stiffness $k_{1}=k_{2}=10 \times 10^{5}$ ]

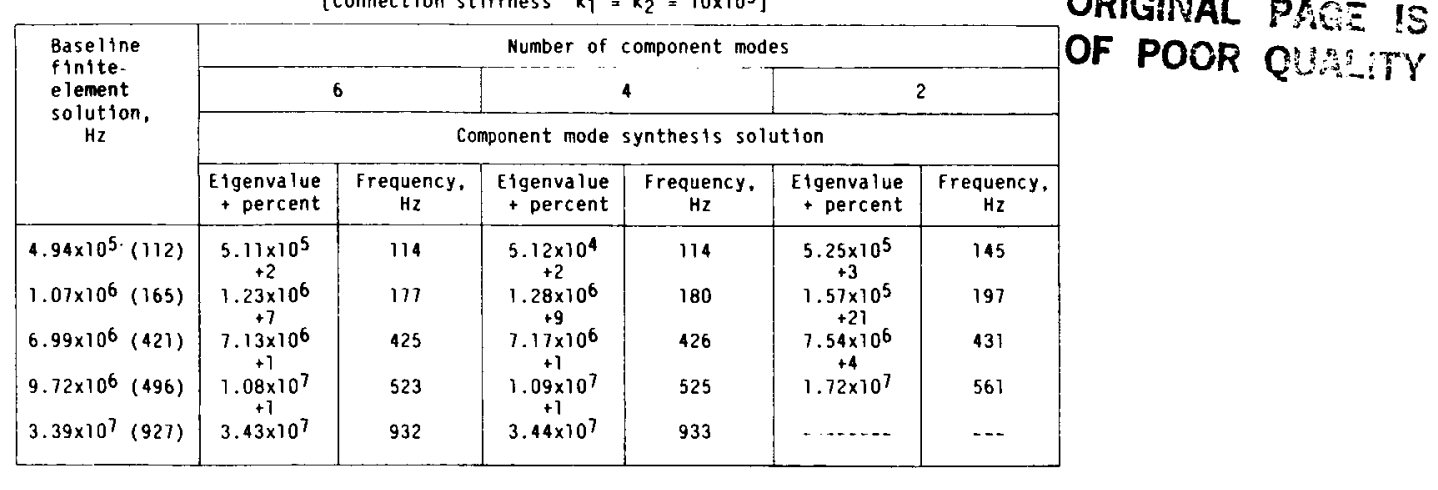

TABLE II. - COMPUTED CONNECTION STIFFNESS

\begin{tabular}{|c|c|c|c|c|c|}
\hline \multirow{4}{*}{$\begin{array}{l}\text { Number of } \\
\text { system } \\
\text { frequencles }\end{array}$} & \multicolumn{5}{|c|}{ Number of component modes } \\
\hline & \multicolumn{2}{|c|}{6} & \multicolumn{2}{|c|}{4} & 2 \\
\hline & \multicolumn{5}{|c|}{ Connection stiffness } \\
\hline & $k_{1}$ & $k_{2}$ & $k_{1}$ & $k_{2}$ & $k_{2}$ \\
\hline \multirow[t]{2}{*}{5} & $6.5 \times 10^{5}$ & $7.4 \times 10^{5}$ & $6.3 \times 10^{5}$ & $7.3 \times 10^{5}$ & \multirow{2}{*}{ (a) } \\
\hline & $3.7 \times 10^{5}$ & $13.1 \times 10^{5}$ & $3.6 \times 10^{5}$ & $12.5 \times 10^{5}$ & \\
\hline \multirow[t]{2}{*}{2} & $6.7 \times 10^{5}$ & $7.4 \times 10^{5}$ & $4.1 \times 10^{5}$ & $10.8 \times 10^{5}$ & \multirow{2}{*}{ (b) } \\
\hline & $3.7 \times 10^{5}$ & $13.4 \times 10^{5}$ & $5.4 \times 10^{5}$ & $8.1 \times 10^{5}$ & \\
\hline
\end{tabular}

a only rour system frequencles avallable.

bsolution does not converge.

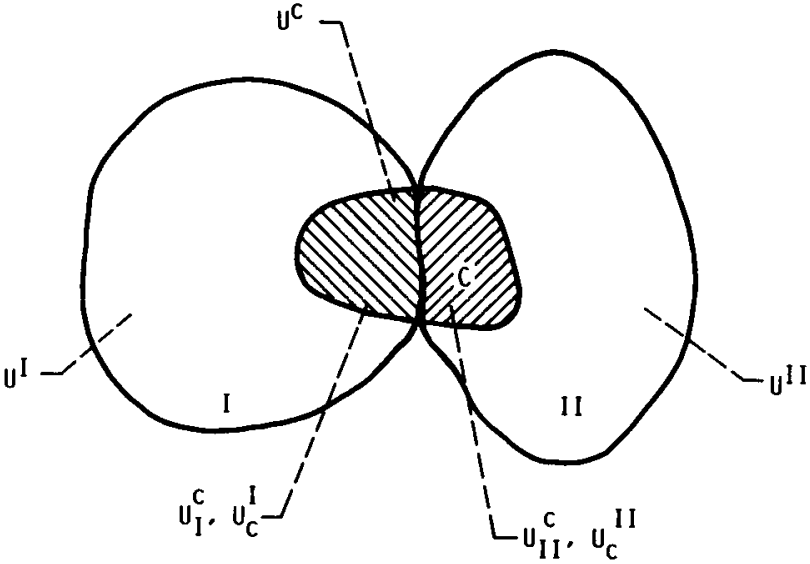

FIGURE 1.- THREE CONPONENT SYSTEM.

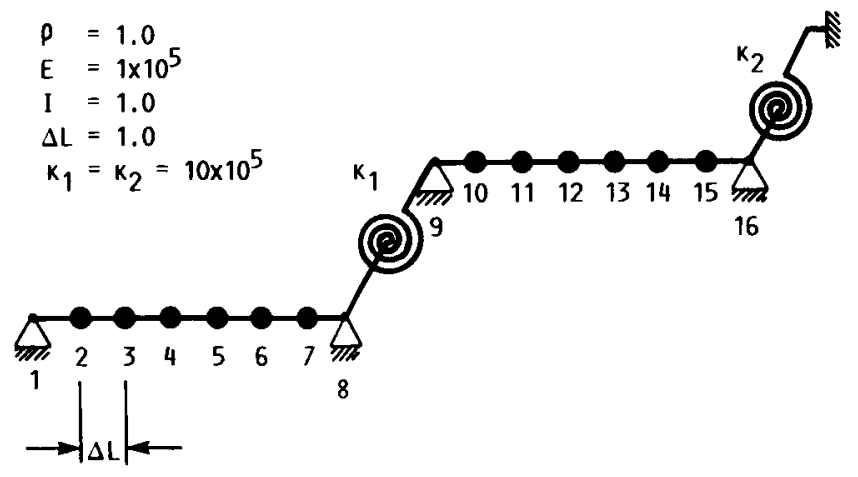

FIGURE 2.- COUPLED SYSTEM (SAMPLE PROBLEM ONE). 


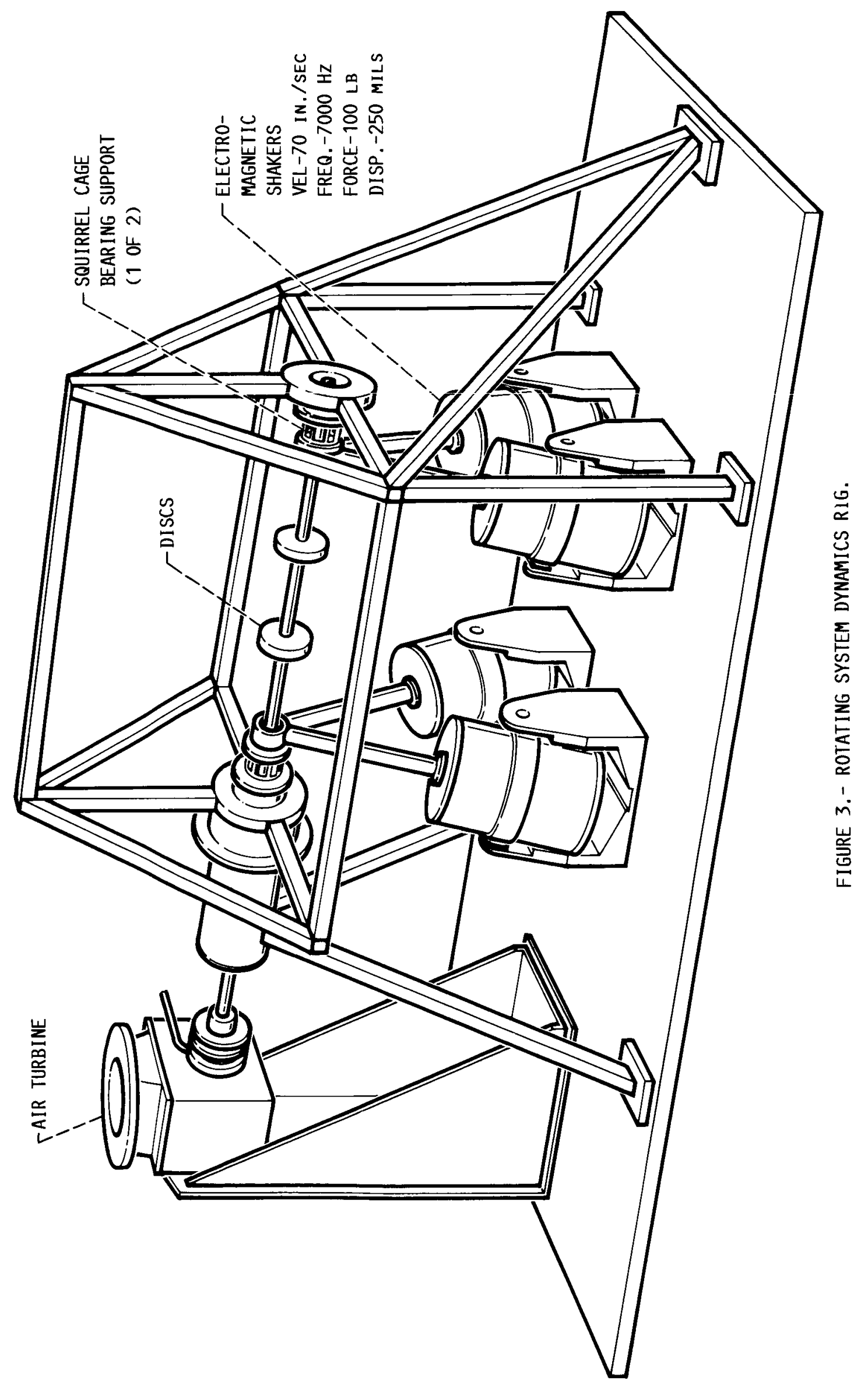




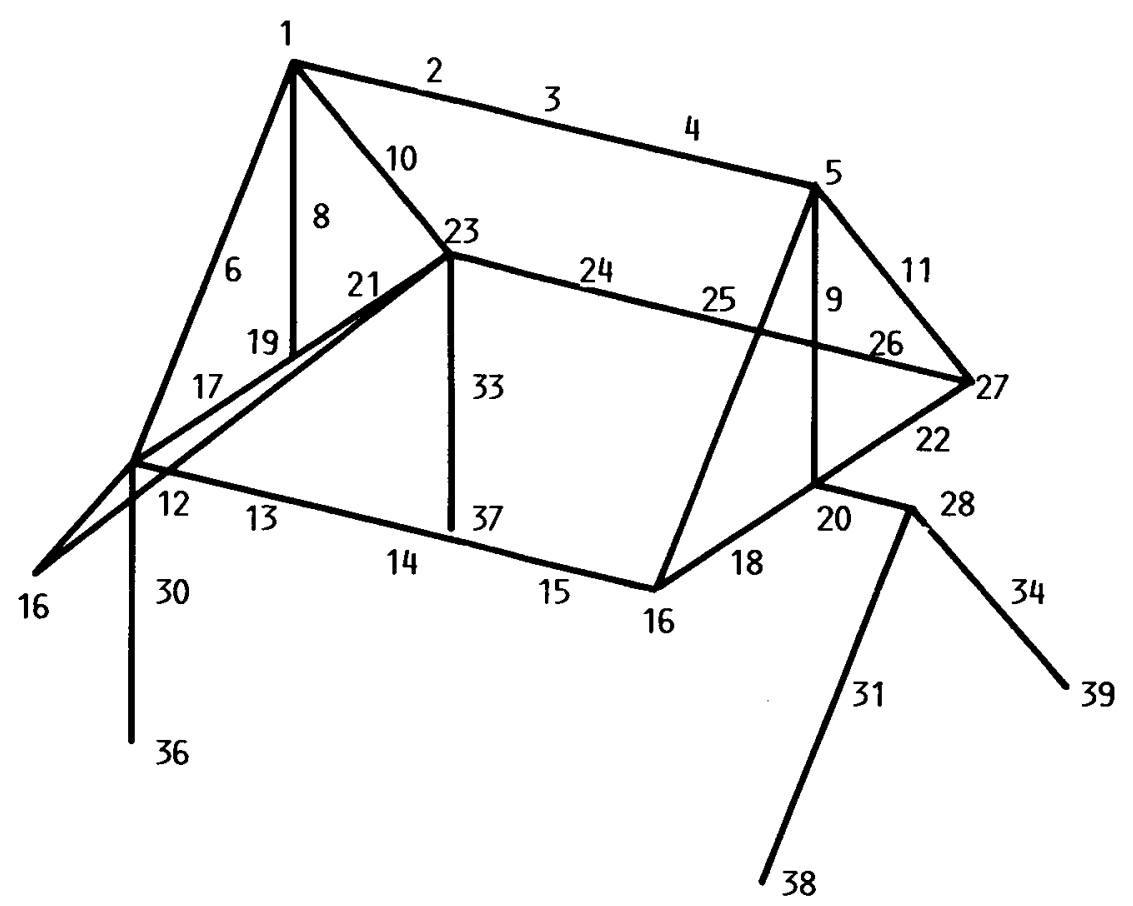

FIGURE 4.- SUPPORT FRAME F.E. MODEL. 


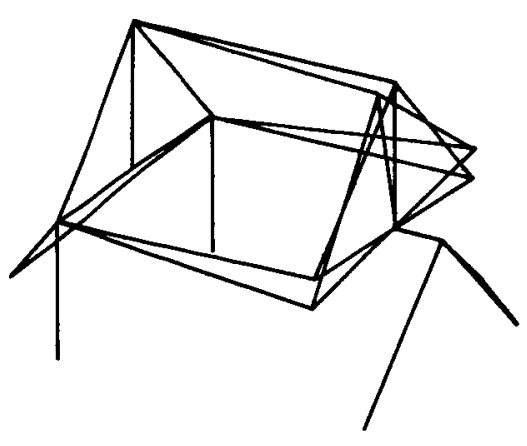

MODE 1: $35 \mathrm{~Hz}$

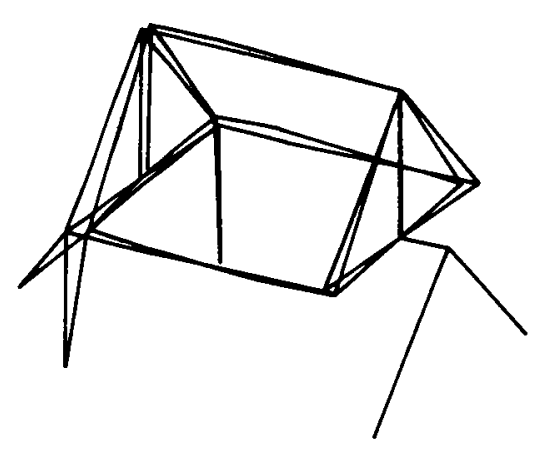

MODE 3: $61 \mathrm{~Hz}$

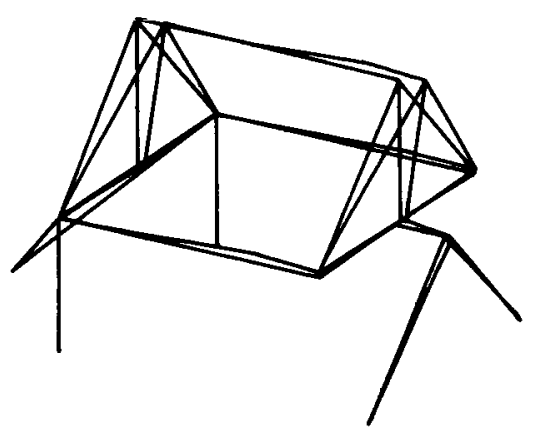

MODE 2: $41 \mathrm{~Hz}$

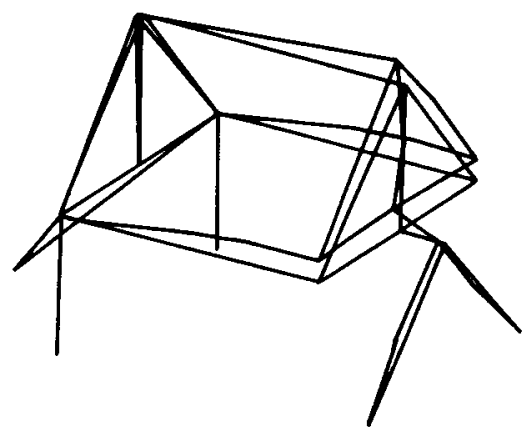

MODE 4: $72 \mathrm{~Hz}$

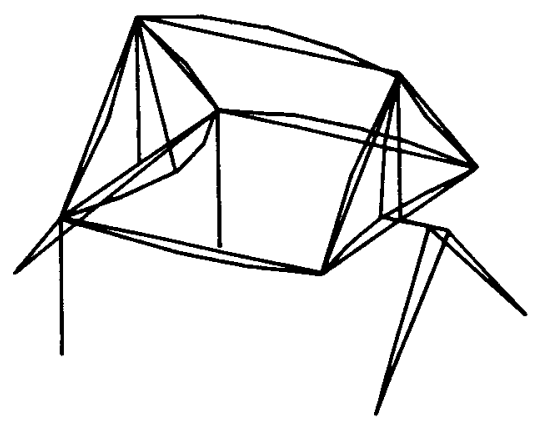

MODE 5: $80 \mathrm{~Hz}$

FIGURE 5.- SUPPORT FRAME MODE SHAPES. 


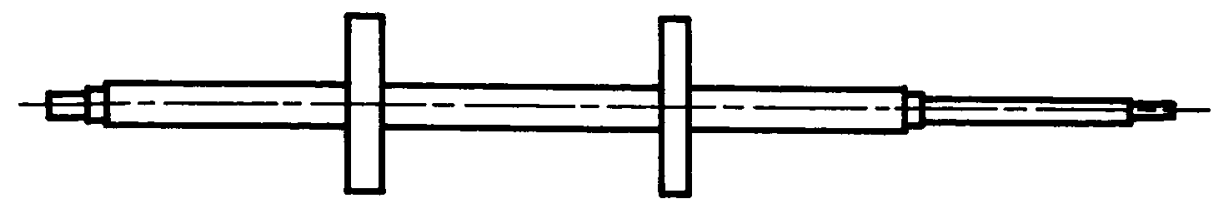

MODE 1: $0 \mathrm{~Hz}$

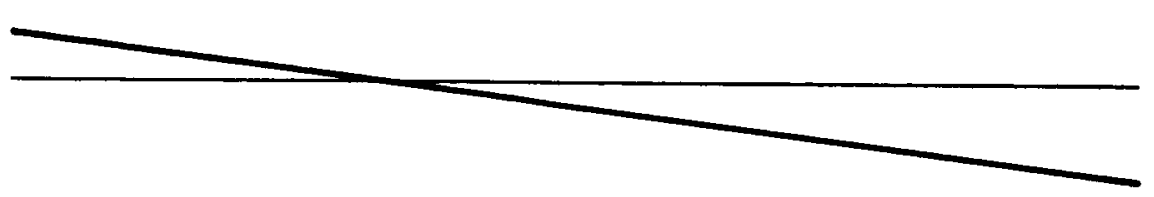

MODE 2: $0 \mathrm{~Hz}$

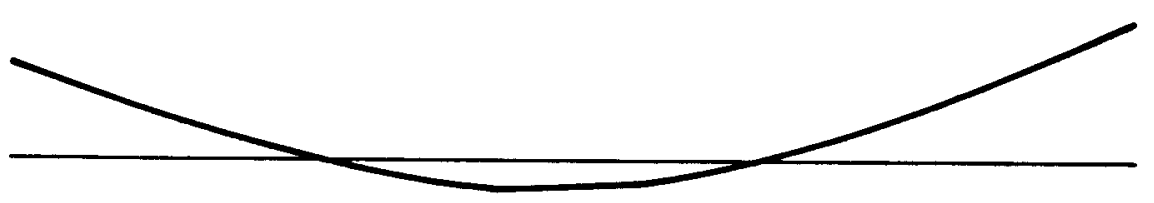

MODE 3: $141 \mathrm{~Hz}$

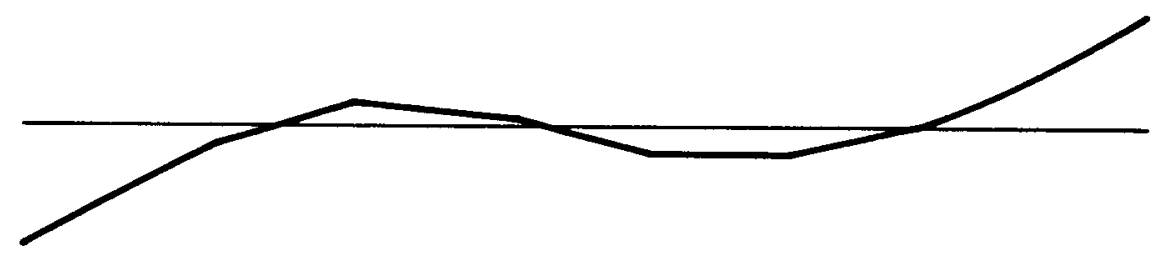

MODE 4: $304 \mathrm{~Hz}$

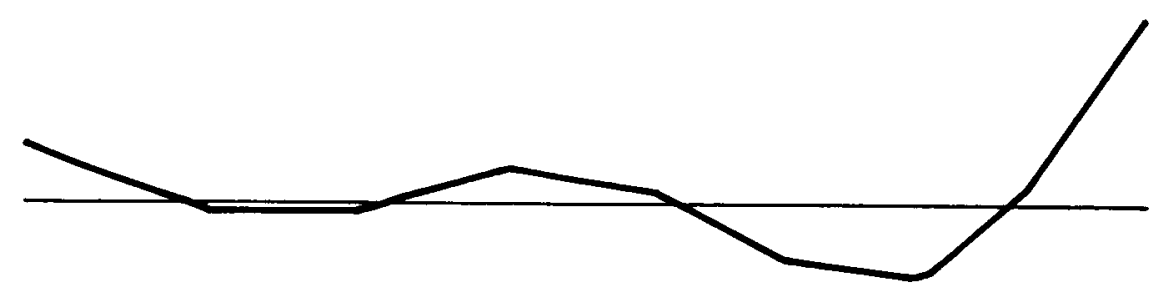

MODE 5: $609 \mathrm{~Hz}$

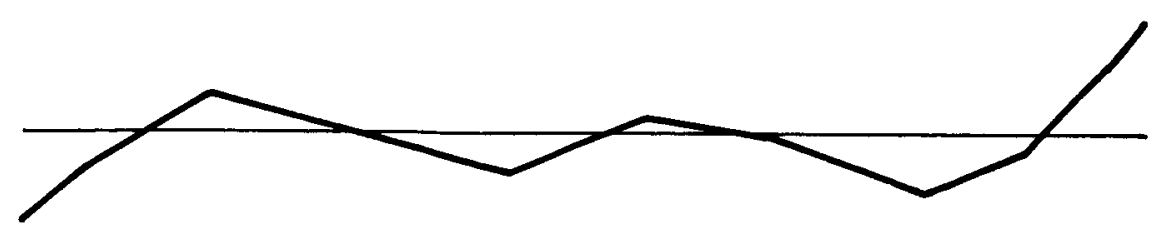

MODE 6: $897 \mathrm{~Hz}$

FIGURE 6. - ROTOR MODE SHAPES. 


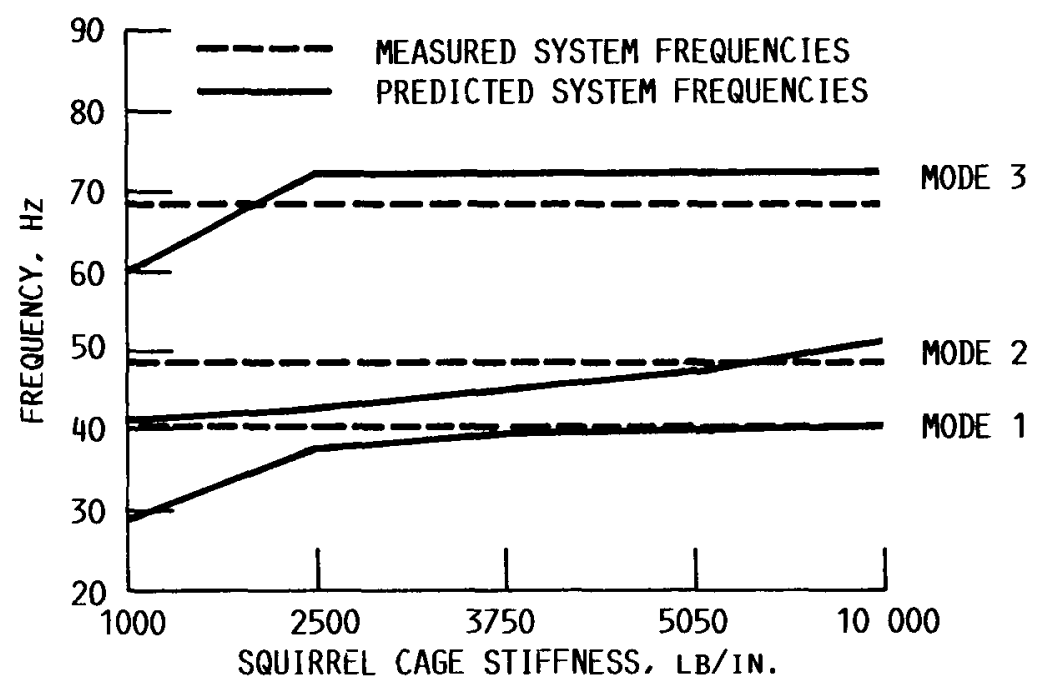

FIGURE 7.- COUPLED FRAME/ROTOR ANALYSIS ( 6 COMPONENT MODES). 


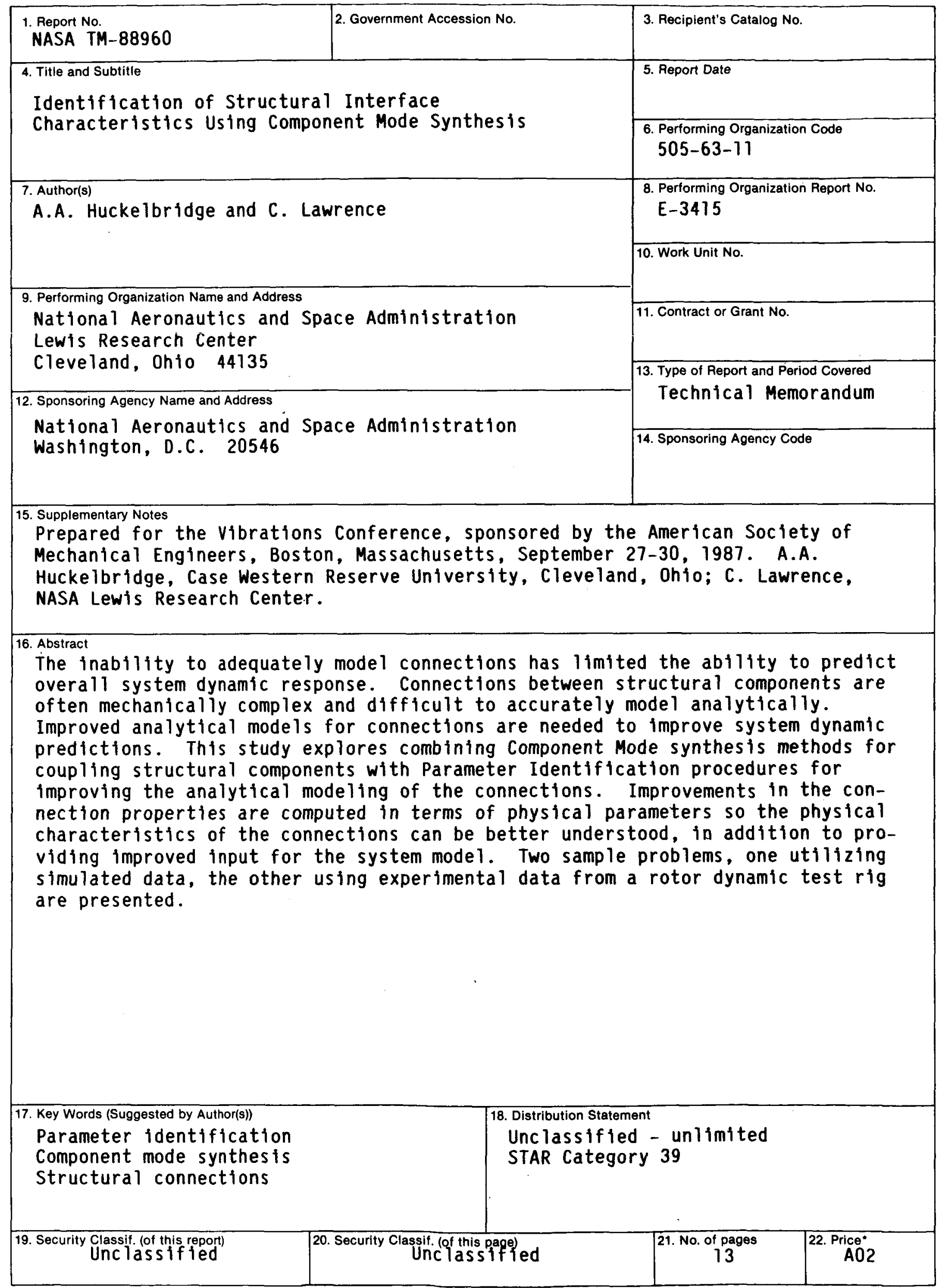

"For sale by the National Technical Information Service, Springfield, Virginia 22161 